\title{
Papel do Farmacêutico no Cuidado a Pessoas com Dermatite Atópica: uma Revisão Sistemática
}

\author{
Iara da Silva Passos, ${ }^{1}$ Maria Caroline Andrade dos Santos, ${ }^{1}$ \\ Ana Caroline Silva Santos, ${ }^{1}$ Izabel Cristina Pereira Rocha, ${ }^{1}$ \\ Elisdete Maria Santos de Jesus, ${ }^{1}$ Chiara Erminia da Rocha, ${ }^{1}$ \\ Giselle de Carvalho Brito ${ }^{2}$
}

\begin{abstract}
RESUMO
Objetivo: Analisar na literatura o papel do farmacêutico no cuidado a pessoas com dermatite atópica. Metodologia: Foi realizada uma revisão sistemática nas bases de dados Medline/PubMed, Lilacs e Web of Science, usando os descritores: dermatitis, eczema, pharmacist. Foram incluídos os artigos escritos em inglês, português e espanhol, que tenham como característica serem estudos sobre o papel do farmacêutico para pessoas com dermatite atópica (DA). Resultados: A pesquisa, inicialmente, identificou 923 resultados nas bases de dados, e, após o processo de seleção, apenas quatro artigos foram incluídos na revisão. Os principais serviços identificados foram o aconselhamento e educação em saúde, que causaram impactos nos resultados clínicos, humanísticos e econômicos. Os resultados clínicos foram informações sobre o tratamento da DA, uso adequado de emolientes e melhoria da compreensão da DA; o econômico resultou na diminuição dos gastos com assistência médica; e o humanístico teve a satisfação dos cuidadores com os vários aspectos do serviço, bem como estavam mais confiantes sobre o tratamento. Conclusão: Apesar dos poucos estudos analisados, pode se observar que o papel do farmacêutico está centralizado no cuidado aos pacientes com DA, prestando serviços de aconselhamento e educação em saúde, contribuindo com informações sobre a doença, sintomas, tratamento farmacológico e não farmacológico e diminuição de problemas quanto ao uso de medicamentos.
\end{abstract}

Palavras-chave: serviços de saúde; intervenções farmacêuticas; dermatite atópica.

\section{ROLE OF THE PHARMACIST IN THE CARE OF PEOPLE WITH ATOPIC DERMATITIS: A SYSTEMATIC REVIEW}

\section{ABSTRACT}

Objective: To analyze in literature the role of pharmacists in the care of people with atopic dermatitis. Methodology: This is a systematic review study in the Medline / PubMed, LILACS and Web of Science databases, using the descriptors: dermatitis, eczema pharmacist. Will be included articles written in English, Portuguese and Spanish, which are characterized as studies on the role of the pharmacist for people with atopic dermatitis. Results: The research initially identified 923 results in the databases, which after the selection process, only four articles were included in the review. The main services identified were health counseling and education, which had an impact on clinical, humanistic and economic results. The main clinical results were information on the treatment of $A D$, adequate use of emollients and improved understanding of $A D$; the economic one, on the other hand, resulted in a decrease in health care expenses; and humanistic, the caregivers were satisfied with the various aspects of the service and were more confident about the treatment. Conclusion: Despite the few studies analyzed, it can be seen that the role of the pharmacist is centralized in the care of patients with AD providing counseling services, health education, contributing with information about the disease, symptoms, pharmacological and non-pharmacological treatment and in reducing problems regarding the use of medicines. Keywords: health services; pharmaceutical interventions; atopic dermatitis.

RECEBIDO EM: 23/9/2020

MODIFICAÇÕES SOLICITADAS EM: 10/2/2021

ACEITO EM: 22/2/2021

\footnotetext{
${ }^{1}$ Universidade Federal de Sergipe

${ }^{2}$ Autora correspondente. Universidade Federal de Sergipe - UFS - Centro de Ciências Biológicas e da Saúde. Av. Marechal s/n, Jardim Rosa Elze. São Cristóvão/SE, Brasil. CEP 49100-000. http://lattes.cnpq.br/0432751118102432. https://orcid. org/0000-0002-3982-2138. gisellecbrito@academico.ufs.br
} 


\section{INTRODUÇÃO}

A dermatite atópica (DA), também conhecida como eczema atópico, é uma doença caracterizada por inflamação crônica e pruriginosa da pele, de caráter recorrente, associada a fatores genéticos, ambientais e alimentares. Normalmente manifesta-se nos primeiros anos de vida, podendo persistir durante a vida adulta e ser também a primeira manifestação de uma doença atópica (GUILHERME et al., 2014). A DA ocorre de maneira cíclica durante a infância. Cerca de 60\% dos pacientes desenvolvem a doença no primeiro ano de vida e $90 \%$ antes dos 5 anos. Somente $25 \%$ dos casos, contudo, persistem na fase adulta (LIMA; NUNES, 2015; ANTUNES et al., 2017).

As pessoas acometidas pela dermatose têm maior probabilidade de desenvolver asma e rinite alérgica em alguma fase da vida, e é frequentemente encontrada em indivíduos com história familiar de DA, porém com manifestação clínica variável (WONG et al., 2017; ANTUNES et al., 2017). Essa doença provoca repercussões psicológicas e sociais, causando inúmeros danos na qualidade de vida dos pacientes, como distúrbios do sono, do humor e evasão escolar, que também são complicações associadas à DA. A família igualmente é afetada, uma vez que tem dificuldades para lidar com as necessidades do paciente, custear seu tratamento e gerenciar um ambiente equilibrado com as pessoas saudáveis (GUILHERME et al., 2014; MENEGUIN; RIBEIRO, 2016).

Os principais objetivos do tratamento da DA envolvem a prevenção de crises, podendo ser de evolução aguda, subaguda ou crônica. A versão aguda tem lesões que começam com as vesículas, que ao se romperem, eliminam o líquido, o que caracteriza a fase subaguda da dermatite. Já na fase crônica, a secreção começa a secar, levando à formação de crostas. Nessa etapa observa-se, também, o aumento da espessura da pele e o reparo e manutenção da barreira cutânea (SBD, 2019; CARVALHO et al., 2017).

O tratamento para DA concentra-se, primeiramente, em rotina rigorosa de cuidados básicos com a pele, utilização de agentes anti-inflamatórios tópicos, corticoides tópicos, anti-histamínicos orais, fototerapia e imunomoduladores sistêmicos. Em casos de infecção por micro-organismos cutâneos oportunistas (bactérias, fungos ou vírus), antibióticos tópicos ou sistêmicos ou antivirais sistêmicos são necessários (CARVALHO et al., 2017; WONG et al., 2017).

Neste contexto, os farmacêuticos podem contribuir para o alcance de resultados positivos da farmacoterapia, educando e aconselhando os pacientes, preparando e motivando-os a seguir os esquemas farmacoterapêuticos para controlar e reduzir a gravidade da doença. A intervenção farmacêutica por meio da educação em saúde é recomendada para todos os pacientes, independentemente da gravidade do quadro, para aumentar a adesão e a eficácia dos tratamentos prescritos. Como os cuidadores estão diretamente envolvidos no cuidado do paciente, intervenções educativas devem ser direcionadas a eles também (CHEONG et al., 2019; YAMADA; NABESHIMA, 2015).

A prevalência da DA, os impactos sociais que provoca, assim como os incômodos para o paciente, requerem a intervenção do farmacêutico não só no apoio e esclarecimento, mas, também, para estabelecer medidas terapêuticas

Editora Unijuí - Revista Contexto \& Saúde - ISSN 2176-7114 - v. 21, n. 43, jul./set. 2021 
de prevenção de recaídas e controle da doença. Na maioria das vezes, o farmacêutico é o profissional de saúde a quem o paciente tem acesso antes de iniciar o tratamento. Assim, a relação farmacêutico-paciente é primordial ao esclarecimento de informações e dúvidas sobre a doença que, muitas vezes, não ficam elucidados na consulta médica (RODRIGUES, 2016).

Apesar do papel potencial dos farmacêuticos no tratamento de problemas de pele, há uma escassez de estudos sobre manejo e desfechos associados ao autocuidado daqueles com condições dermatológicas, especialmente em DA (HAMMARSTRÖM; WESSLING; NILSSON, 1995; CHEONG et al., 2019, YAMADA; NABESHIMA, 2015). O estudo de Tucker et al. (2017) indica a necessidade de maior formação relacionada à avaliação farmacêutica em dermatologia. Enquanto Cheong et al. (2019), revelaram o impacto de um serviço de aconselhamento farmacêutico para dermatite quanto à melhora do conhecimento dos cuidadores no tratamento da dermatite atópica pediátrica.

É necessário, entretanto, investigar na literatura quais as funções que o farmacêutico vem desempenhando ante a esta condição clínica, o que poderá auxiliar desde o processo de formação profissional até o fornecimento de subsídios para que outros farmacêuticos possam reorientar sua prática profissional quando se depararem com pessoas com dermatite atópica. Assim, em virtude do que foi exposto, o objetivo do presente estudo foi analisar o papel do farmacêutico no cuidado a pessoas com dermatite atópica.

\section{METODOLOGIA}

Trata-se de uma revisão sistemática segundo a metodologia descrita por Preferred Reporting Items for Systematic Reviews and Meta-Analyses (Prisma) do tipo experiencial (GALVÃO; PANSANI; HARRAD, 2015). Os artigos que foram identificados estão indexados nas bases de dados Web of Science e Biblioteca Virtual em Saúde (BVS), que contempla duas outras bases de dados: Literatura Latino-Americana e do Caribe em Ciências da Saúde (Lilacs) e Medical Literature Analysis and Retrieval System On-line/PubMed (Medline).

Foram utilizados os seguintes Descritores em Ciências da Saúde (Decs) e no Medical Subject Headings (MeSH), e seus sinônimos nos idiomas inglês, português e espanhol em diferentes combinações: dermatitis; eczema; pharmacist, que relatem o papel do farmacêutico para pessoas com dermatite atópica. Para as buscas, os descritores foram escritos no idioma inglês e utilizados isoladamente e em combinação com o auxílio dos operadores booleanos "AND" e "OR", de forma conveniente e de acordo com as peculiaridades de cada base de dados pesquisada.

A seleção dos estudos aconteceu entre 8 de outubro de 2019 e 16 de outubro de 2019, com base nos critérios de inclusão estabelecidos: 1) Inicialmente houve a seleção dos estudos mediante a leitura e a análise dos títulos e resumos de todos os artigos identificados; 2) Na sequência do refinamento ocorreu a leitura na íntegra dos estudos selecionados, a qual possibilitou que outros textos que não atendessem à proposta da revisão fossem excluídos. Ainda, as referências dos estudos incluídos foram analisadas a fim de identificar outros artigos

Editora Unijuí - Revista Contexto \& Saúde - ISSN 2176-7114 - v. 21, n. 43, jul./set. 2021 
relevantes que podem ser adicionados à amostra final; 3) Por fim, as variáveis de interesse dos artigos foram sintetizadas em uma planilha para orientar as análises descritivas e críticas dos estudos selecionados. Foram excluídos artigos de revisão sistemática, relatórios de pesquisa, teses, dissertações, livros, capítulos de livros, editoriais, artigos de opinião de especialistas e trabalhos publicados em anais de eventos científicos.

A pesquisa, inicialmente, identificou 923 títulos nas bases de dados, após exclusão dos artigos duplicados e leitura dos títulos e resumos; 27 artigos foram selecionados para a triagem de texto completo, dos quais apenas quatro preencheram os critérios de inclusão. O fluxograma do processo de seleção dos estudos de acordo com os critérios de inclusão é mostrado na Figura 1.

Figura 1 - Fluxograma de seleção dos artigos incluídos na revisão sistemática
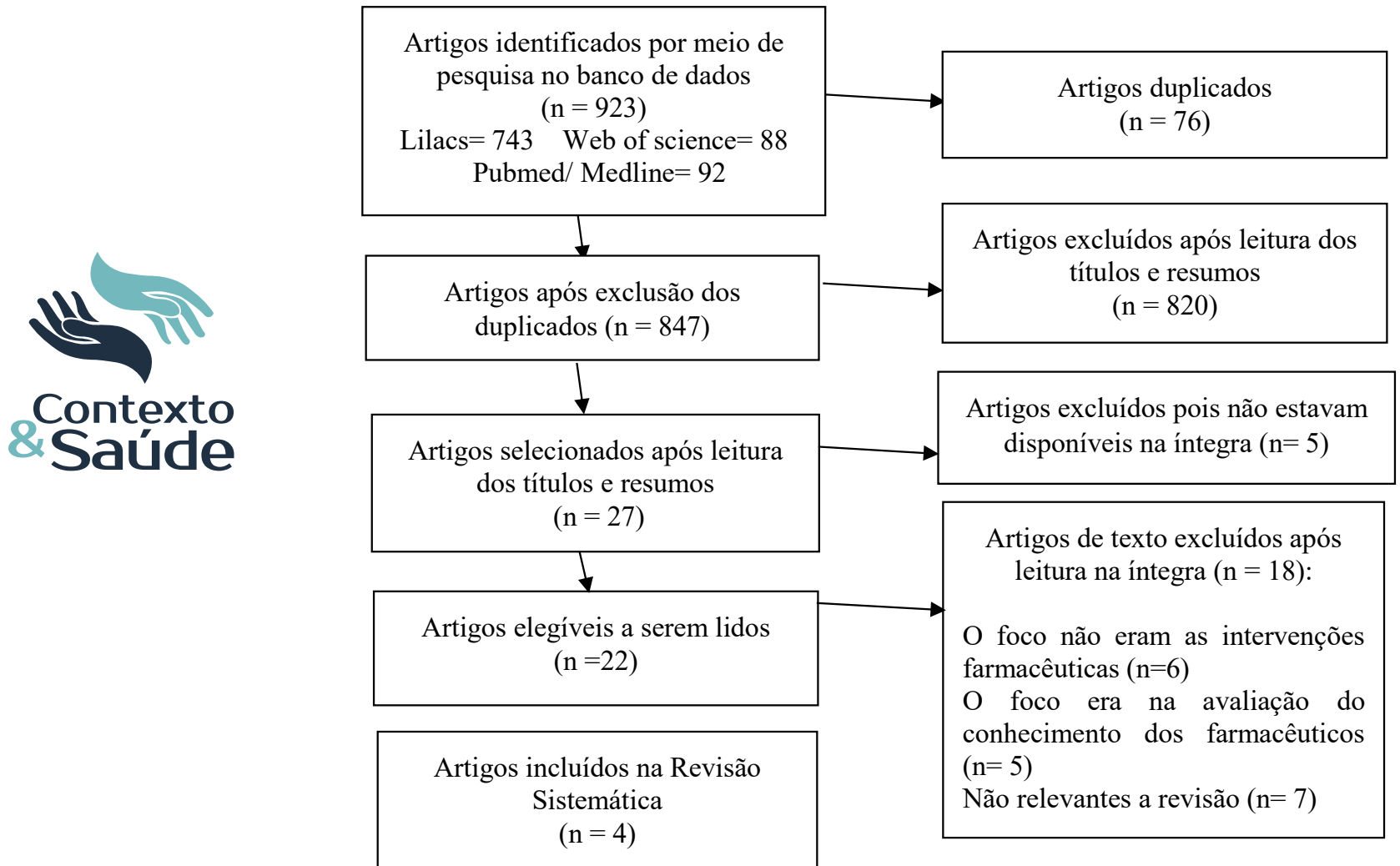

Fonte: As autoras.

\section{RESULTADOS}

Quanto ao cenário, os estudos foram desenvolvidos em ambulatórios hospitalares (CHEONG et al., 2019; FARRUGIA et al., 2017) e em farmácias comunitárias (HAMMARSTRÖM; WESSLING; NILSSON, 1995; KOSTER et al., 2019). Os delineamentos metodológicos declarados nos estudos foram: estudo observacional prospectivo (CHEONG et al., 2019), transversal multicêntrico (FARRUGIA et al., 2017), estudo qualitativo (KOSTER et al., 2019) e um não especificou o desenho metodológico (HAMMARSTRÖM; WESSLING; NILSSON, 1995) (Tabela 1). 
Os estudos foram realizados na Suécia, Cingapura, Austrália e Países Baixos. Esses foram publicadas em inglês entre 1995 e 2019, com tempo de duração variável entre quatro meses (KOSTER et al., 2019), sete meses (CHEONG et al., 2019), 13 meses (FARRUGIA et al., 2017) e 36 meses (HAMMARSTRÖM; WESSLING; NILSSON, 1995) (Tabela 1).

No que diz respeito à amostra estudada, Hammarström, Wessling e Nilsson (1995) envolveram todas as faixas etárias, porém não avaliaram o tamanho da população do estudo, pois era direcionado para pacientes e profissionais da saúde. Já Cheong et al. (2019) apresentaram uma amostra de 32 cuidadores com idade variável de 21 a 80 anos. Farrugia et al. (2017) tiveram uma amostra de 201 participantes, incluindo pacientes dermatológicos maiores de 18 anos e cuidadores de pacientes pediátricos com idade $<18$ anos. O estudo de Koster et al. (2019) teve uma amostra de 29 cuidadores de crianças de zero a 12 anos (Tabela 1).

Tabela 1 - Características gerais dos estudos incluídos na revisão sistemática

\begin{tabular}{|c|c|c|c|c|c|}
\hline $\begin{array}{c}\text { Autor/ano/ } \\
\text { local }\end{array}$ & $\begin{array}{c}\text { População-alvo/ } \\
\text { faixa etária/Ce- } \\
\text { nário }\end{array}$ & Objetivo do estudo & $\begin{array}{l}\text { Tipo de es- } \\
\text { tudo }\end{array}$ & $\begin{array}{c}\text { Duração } \\
\text { do estudo }\end{array}$ & $\begin{array}{c}\text { Tamanho } \\
\text { da amostra }\end{array}$ \\
\hline $\begin{array}{l}\text { Hammars- } \\
\text { tröm; Wess- } \\
\text { ling; Nilsson } \\
\text { (1995), } \\
\text { Suécia }\end{array}$ & $\begin{array}{c}\text { Pacientes e } \\
\text { profissionais de } \\
\text { saúde/todas/Far- } \\
\text { mácia }\end{array}$ & $\begin{array}{l}\text { Aumentar a cons- } \\
\text { cientização, adesão } \\
\text { e adequação do } \\
\text { tratamento de do- } \\
\text { enças de pele em } \\
\text { pacientes atendidos } \\
\text { em farmácias. }\end{array}$ & Não declarado & 36 meses & $\begin{array}{l}\text { Não ava- } \\
\text { liado }\end{array}$ \\
\hline $\begin{array}{l}\text { Cheong et } \\
\text { al. (2019), } \\
\text { Cingapura }\end{array}$ & $\begin{array}{l}\text { Cuidadores/21 a } \\
80 \text { anos/Ambula- } \\
\text { tório hospitalar }\end{array}$ & $\begin{array}{l}\text { Avaliar o impacto } \\
\text { de um serviço de } \\
\text { aconselhamento de } \\
\text { dermatite liderado } \\
\text { por farmacêuticos } \\
\text { na melhoria do } \\
\text { conhecimento dos } \\
\text { cuidadores. }\end{array}$ & $\begin{array}{c}\text { Observacional } \\
\text { prospectivo }\end{array}$ & 7 meses & 32 \\
\hline $\begin{array}{l}\text { Farrugia et } \\
\text { al. (2017), } \\
\text { Austrália }\end{array}$ & $\begin{array}{c}\text { Cuidadores e } \\
\text { adolescentes/ } \leq 18 \\
\text { anos/Ambulató- } \\
\text { rio hospitalar }\end{array}$ & $\begin{array}{l}\text { Avaliar os conselhos } \\
\text { e comportamentos } \\
\text { do farmacêutico e } \\
\text { clínico geral (GP), } \\
\text { relacionados e rela- } \\
\text { tados por pacientes } \\
\text { e pais de pacientes } \\
\text { que usam corticoste- } \\
\text { roides tópicos. }\end{array}$ & $\begin{array}{l}\text { Transversal } \\
\text { multicêntrico }\end{array}$ & 13 meses & 201 \\
\hline $\begin{array}{l}\text { Koster et al. } \\
\text { (2019), Paí- } \\
\text { ses Baixos }\end{array}$ & $\begin{array}{c}\text { Cuidadores/Zero } \\
\text { a } 12 \text { anos/Far- } \\
\text { mácia }\end{array}$ & $\begin{array}{c}\text { Explorar a perspecti- } \\
\text { va dos funcionários } \\
\text { da farmácia e pais } \\
\text { sobre o tratamento } \\
\text { de pacientes jovens } \\
\text { com DA no Países } \\
\text { Baixos. } \\
\end{array}$ & Qualitativo & 4 meses & 29 \\
\hline
\end{tabular}

Fonte: Dados da pesquisa. 
Os serviços farmacêuticos reportados nos estudos foram o aconselhamento farmacêutico (HAMMARSTRÖM; WESSLING; NILSSON, 1995; CHEONG et al., 2018; FARRUGIA et al., 2017; KOSTER et al., 2019) e educação em saúde (HAMMARSTRÖM; WESSLING; NILSSON, 1995), que causaram impacto nos resultados clínicos (HAMMARSTRÖM; WESSLING; NILSSON, 1995; CHEONG et al., 2018; FARRUGIA et al., 2017; KOSTER et al., 2019) humanísticos (HAMMARSTRÖM; WESSLING; NILSSON, 1995; CHEONG et al., 2018; FARRUGIA et al., 2017; KOSTER et al., 2019) e econômicos (HAMMARSTRÖM; WESSLING; NILSSON, 1995) que estão descritos na Tabela 2.

Tabela 2 - Características dos estudos incluídos na revisão sistemática quanto à colaboração de profissionais, conclusões e limitações

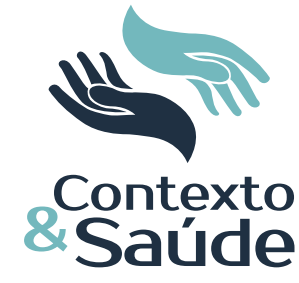

\begin{tabular}{|c|c|c|c|}
\hline Autor/ano & $\begin{array}{l}\text { Colaboração com } \\
\text { outros profissio- } \\
\text { nais }\end{array}$ & Principais Conclusões & Limitações \\
\hline $\begin{array}{l}\text { Hammarström; } \\
\text { Wessling; Nils- } \\
\text { son (1995) }\end{array}$ & $\begin{array}{l}\text { Os coordenadores } \\
\text { da campanha } \\
\text { "ano da pele" } \\
\text { apresentaram e } \\
\text { discutiram sobre } \\
\text { a campanha com } \\
\text { seus dermatolo- } \\
\text { gistas e médicos } \\
\text { de clínica geral. }\end{array}$ & $\begin{array}{l}\text { Os pacientes aprenderam a im- } \\
\text { portância do uso contínuo de } \\
\text { emolientes no tratamento da } \\
\text { DA. Houve uma resposta posi- } \\
\text { tiva à campanha. Os médicos } \\
\text { estão capacitados para informar } \\
\text { aos pacientes sobre o uso de } \\
\text { emolientes e a prescrição des- } \\
\text { ses produtos. }\end{array}$ & $\begin{array}{l}\text { Tempo do estudo, posto que uma ava- } \\
\text { liação de um ano poderia ser comple- } \\
\text { xa e cara. Dados comparáveis sobre o } \\
\text { resultado do paciente como resultado } \\
\text { da campanha não estão disponíveis. } \\
\text { Os dados não foram compilados em } \\
\text { âmbito nacional. }\end{array}$ \\
\hline $\begin{array}{c}\text { Cheong et al. } \\
\text { (2019) }\end{array}$ & $\begin{array}{l}\text { Os materiais } \\
\text { educacionais } \\
\text { foram revisados } \\
\text { por uma equipe } \\
\text { de farmacêuticos } \\
\text { e dermatologistas } \\
\text { e forneceram } \\
\text { treinamento. }\end{array}$ & $\begin{array}{l}\text { O serviço de aconselhamento } \\
\text { farmacêuticos tem um impacto } \\
\text { positivo no conhecimento dos } \\
\text { cuidadores e no gerenciamento } \\
\text { da condição do filho, melhoran- } \\
\text { do a conformidade e o cuidado } \\
\text { da condição do paciente. }\end{array}$ & $\begin{array}{l}\text { Os resultados clínicos dos pacientes } \\
\text { não foram estudados, o que teria } \\
\text { aumentado o impacto do papel do } \\
\text { aconselhamento farmacêutico no tra- } \\
\text { tamento da DA. Outra limitação foi o } \\
\text { pequeno número de sujeitos recruta- } \\
\text { dos para o estudo em razão do curto } \\
\text { período de estudo. Além disso, alguns } \\
\text { cuidadores relutaram em participar } \\
\text { pela restrições de tempo. }\end{array}$ \\
\hline $\begin{array}{l}\text { Farrugia et al. } \\
\qquad(2017)\end{array}$ & $\begin{array}{l}\text { Não houve cola- } \\
\text { borador. }\end{array}$ & $\begin{array}{l}\text { A adesão à terapia prolongada } \\
\text { com corticosteroides tópicos é } \\
\text { baixa, enquanto pacientes e as } \\
\text { crenças dos pais sobre os "ris- } \\
\text { cos" dos corticosteroides tó- } \\
\text { picos são comuns. Mensagens } \\
\text { incorretas sobre os "perigos" } \\
\text { do uso prolongado de corticos- } \\
\text { teroides tópicos estão sendo } \\
\text { recebidas pela maioria dos pa- } \\
\text { cientes e pais de forma consis- } \\
\text { tente por profissionais de saúde } \\
\text { confiáveis, como farmacêuticos } \\
\text { e clínicos gerais }\end{array}$ & $\begin{array}{l}\text { O estudo não consegue colocar as } \\
\text { respostas dos entrevistados no con- } \\
\text { texto de seus relacionamentos com } \\
\text { médicos e farmacêuticos. Por exem- } \\
\text { plo, um paciente que não tem um } \\
\text { clínico geral regular pode ser aconse- } \\
\text { Ihado de maneira diferente a um pa- } \\
\text { ciente que teve um relacionamento } \\
\text { de longo prazo com um clínico geral } \\
\text { confiável. Incapacidade de conside- } \\
\text { rar a personalidade e outros fatores } \\
\text { pessoais, que podem contribuir para } \\
\text { comportamentos e atitudes dos pa- } \\
\text { cientes e dos pais. }\end{array}$ \\
\hline $\begin{array}{l}\text { Koster et al. } \\
\text { (2019) }\end{array}$ & $\begin{array}{l}\text { Reunião com } \\
\text { clínico geral e } \\
\text { pediatra para } \\
\text { determinar as } \\
\text { opiniões dos es- } \\
\text { pecialistas sobre } \\
\text { o tratamento da } \\
\text { dermatite atópica } \\
\text { e suas sugestões } \\
\text { para áreas de } \\
\text { melhoria. }\end{array}$ & $\begin{array}{l}\text { A percepção dos pais sobre o } \\
\text { corticosteroides tópicos pode } \\
\text { influenciar negativamente re- } \\
\text { sultados do tratamento. O pes- } \\
\text { soal da farmácia tem um papel } \\
\text { importante: informar os pais de } \\
\text { crianças com DA sobre a uso de } \\
\text { corticosteroides tópicos e emo- } \\
\text { lientes. }\end{array}$ & $\begin{array}{l}\text { O estudo pode ter sido sujeito a al- } \\
\text { gum grau de viés de seleção, pois os } \\
\text { pais que apresentam problemas ou } \\
\text { têm um filho com doença mais grave } \\
\text { podem ter maior probabilidade de } \\
\text { participar. Além disso, o estudo ex- } \\
\text { plorou as opiniões dos pais sobre o } \\
\text { impacto da DA no filho, o que pode } \\
\text { diferir do impacto real que a criança } \\
\text { experimenta. As entrevistas foram } \\
\text { codificada por um pesquisador, e é } \\
\text { possível que algumas informações } \\
\text { não tenham sido codificadas correta- } \\
\text { mente. }\end{array}$ \\
\hline
\end{tabular}

Fonte: Dados da pesquisa. 
Quanto à classificação das intervenções, um estudo foi classificado como comportamental (FARRUGIA et al., 2017), um foi técnico (FARRUGIA et al., 2017), um como educacional (HAMMARSTRÖM; WESSLING; NILSSON, 1995) e outro foi combinado, utilizando mais de uma das três categorias acima (técnicas, educacionais, comportamentais) (CHEONG et al., 2019). As estratégias às intervenções estão descritas na Tabela 3.

No que se refere às intervenções, destaca-se programas com o objetivo de educar os médicos, pacientes e cuidadores, relacionados à DA (CHEONG et al., 2019; HAMMARSTRÖM; WESSLING; NILSSON, 1995). Com isso, os farmacêuticos orientam sobre quais os melhores emolientes para cada tipo de pele e a administração de medicamentos, minimizando efeitos colaterais/toxicidade, além de falar sobre os medos e mitos com relação aos corticosteroides tópicos e realizar a otimização do conhecimento sobre a dermatite e seu tratamento, oferecendo educação e apoio, a fim de melhorar o resultado e qualidade de vida dos pacientes (Tabela 3).

Tabela 3 - Características das intervenções farmacêuticas dos estudos incluídos na revisão sistemática

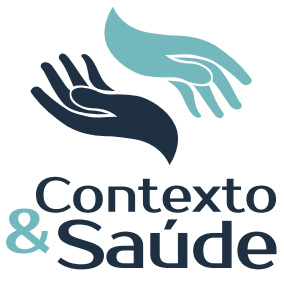

\begin{tabular}{|c|c|c|c|c|c|c|}
\hline \multirow[t]{2}{*}{ Autor/ano } & \multirow{2}{*}{$\begin{array}{c}\text { Serviços } \\
\text { desenvol- } \\
\text { vidos } \\
\end{array}$} & \multicolumn{4}{|c|}{ Intervenções farmacêuticas } & \multirow{2}{*}{ Resultados } \\
\hline & & $\begin{array}{l}\text { Comporta- } \\
\text { mental }\end{array}$ & Técnico & Educacional & $\begin{array}{l}\text { Combina- } \\
\text { ção }\end{array}$ & \\
\hline $\begin{array}{l}\text { Hammars- } \\
\text { tröm; Wess- } \\
\text { ling; Nilsson } \\
\text { (1995) }\end{array}$ & $\begin{array}{l}\text { Campanha } \\
\text { educacional }\end{array}$ & NR & NR & $\begin{array}{l}\text { Conselhos sobre } \\
\text { problemas de pele } \\
\text { e um conjunto de } \\
\text { desenhos a ser em- } \\
\text { pregado para infor- } \\
\text { mações e educação } \\
\text { do paciente sobre o } \\
\text { uso e a aplicação de } \\
\text { pomadas. Uma caixa } \\
\text { com diferentes tipos } \\
\text { de veículos dermato- } \\
\text { lógicos e uma apre- } \\
\text { sentação escrita dos } \\
\text { veículos e material } \\
\text { de palestra sobre dez } \\
\text { temas diferentes }\end{array}$ & NR & $\begin{array}{l}\text { Clínico: Os pacientes apren- } \\
\text { deram a importância do uso } \\
\text { contínuo de emolientes no } \\
\text { tratamento de DA; aumento } \\
\text { da prescrição de emolientes } \\
\text { como complemento e pres- } \\
\text { crição de corticosteroides; } \\
\text { o tratamento das doenças } \\
\text { aumentou substancialmen- } \\
\text { te e houve maior conscienti- } \\
\text { zação, adesão e adequação } \\
\text { do tratamento. } \\
\text { Econômico: Redução de } \\
\text { custos de, pelo menos, US\$ } \\
15 \text { milhões ou 5\%-10\% } \\
\text { dos custos com assistência } \\
\text { médica para desordens da } \\
\text { pele. } \\
\text { Humanístico: Não avaliado }\end{array}$ \\
\hline $\begin{array}{c}\text { Cheong et al. } \\
\text { (2019) }\end{array}$ & $\begin{array}{l}\text { Aconselha- } \\
\text { mento }\end{array}$ & NR & NR & NR & $\begin{array}{l}\text { Gatilhos da } \\
\text { DA, o mane- } \\
\text { jo farmaco- } \\
\text { lógico e não } \\
\text { farmacoló- } \\
\text { gico e o uso } \\
\text { apropriado } \\
\text { de prepara- } \\
\text { ções tópicas }\end{array}$ & $\begin{array}{l}\text { Clínico: O aconselhamento } \\
\text { farmacêutico melhorou a } \\
\text { compreensão dos cuidado- } \\
\text { res sobre o uso de medica- } \\
\text { mentos. O aconselhamento } \\
\text { farmacêutico melhorou o } \\
\text { conhecimento dos pacien- } \\
\text { tes e dos pais sobre várias } \\
\text { condições crônicas } \\
\text { Econômico: não avaliado } \\
\text { Humanístico: Os cuidadores } \\
\text { ficaram bastante satisfeitos } \\
\text { com os vários aspectos do } \\
\text { serviço e estavam mais con- } \\
\text { fiantes sobre o tratamento } \\
\text { do filho; cuidadores que } \\
\text { abrigam mitos relacionados } \\
\text { à fobia a esteroide podem } \\
\text { se beneficiar do serviço } \\
\text { dedicado sobre este tópico }\end{array}$ \\
\hline
\end{tabular}




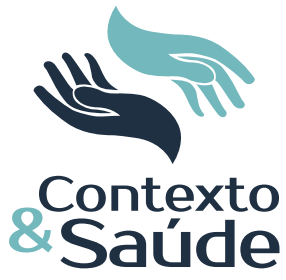

\begin{tabular}{|c|c|c|c|c|c|c|}
\hline $\begin{array}{l}\text { Farrugia et al. } \\
\text { (2017) }\end{array}$ & $\begin{array}{l}\text { Aconselha- } \\
\text { mento }\end{array}$ & $\begin{array}{l}\text { Mensagens } \\
\text { sobre o } \\
\text { corticosteroi- } \\
\text { des tópicos } \\
\text { recebido de } \\
\text { farmacêuticos } \\
\text { comunitários, } \\
\text { clínicos gerais, } \\
\text { familiares/ } \\
\text { amigos e da } \\
\text { Internet. }\end{array}$ & $\begin{array}{l}\text { Avaliada a } \\
\text { adesão ao } \\
\text { tratamento com } \\
\text { corticosteroides } \\
\text { tópicos, moti- } \\
\text { vação e razões } \\
\text { para a não ade- } \\
\text { são; crenças a } \\
\text { respeito do uso } \\
\text { e segurança do } \\
\text { corticosteroide } \\
\text { tópico; men- } \\
\text { sagens sobre o } \\
\text { corticosteroides } \\
\text { tópicos recebido } \\
\text { de farmacêu- } \\
\text { ticos comuni- } \\
\text { tários, clínicos } \\
\text { gerais, familia- } \\
\text { res/amigos e } \\
\text { da Internet; e } \\
\text { experiência de } \\
\text { clínicos gerais e } \\
\text { aconselhamento } \\
\text { farmacêutico. }\end{array}$ & NR & NR & $\begin{array}{l}\text { Clínico: Identificação da } \\
\text { baixa adesão à terapia } \\
\text { prolongada com corticoste- } \\
\text { roides tópicos em razão da } \\
\text { preocupação com os efeitos } \\
\text { a longo prazo } \\
\text { Econômico: não avaliado } \\
\text { Humanístico Mensagens } \\
\text { incorretas sobre os “pe- } \\
\text { rigos” do uso prolongado } \\
\text { de corticosteroides tópicos } \\
\text { estão sendo recebidas pela } \\
\text { maioria dos pacientes e } \\
\text { pais de forma consistente } \\
\text { por profissionais de saúde } \\
\text { confiáveis, como farma- } \\
\text { cêuticos e clínicos gerais. } \\
\text { Pacientes e pais relatam ter } \\
\text { experiências negativas de } \\
\text { aconselhamento farmacêu- } \\
\text { tico e clínicos gerais. }\end{array}$ \\
\hline $\begin{array}{c}\text { Koster et al. } \\
\text { (2019) }\end{array}$ & $\begin{array}{l}\text { Aconselha- } \\
\text { mento }\end{array}$ & NR & NR & $\begin{array}{l}\text { Informações sobre } \\
\text { uso dos diferentes } \\
\text { cremes e informa- } \\
\text { ções sobre efeitos } \\
\text { adversos, ação } \\
\text { farmacológico, não } \\
\text { farmacológica e } \\
\text { estilo de vida. }\end{array}$ & NR & $\begin{array}{l}\text { Clínico: A percepção dos } \\
\text { pais sobre o corticosteroide } \\
\text { tópico influencia negati- } \\
\text { vamente os resultados do } \\
\text { tratamento; informou aos } \\
\text { pais os prós e contras dos } \\
\text { corticosteroides tópicos; } \\
\text { uso adequado de corticos- } \\
\text { teroides em crianças e na } \\
\text { escolha do emoliente ade- } \\
\text { quado para o tratamento de } \\
\text { crianças com dermatite } \\
\text { Econômico: não avaliado } \\
\text { Humanístico: não avaliado }\end{array}$ \\
\hline
\end{tabular}

Fonte: Dados da pesquisa. NR= Não relatado.

\section{DISCUSSÃO}

Os estudos foram realizados em ambulatórios ou farmácias. A inserção do farmacêutico nestes cenários propicia confiança ao paciente, responsabilizando-o pelos problemas enfrentados por este em relação às suas condições de saúde e ao seu tratamento (BRASIL, 2014). Além da dispensação de medicamentos e produtos para a saúde, busca-se, atualmente, o desenvolvimento de serviços clínicos providos por farmacêuticos (MENDONÇA; FERREIRA; LA ROCQUE, 2017).

Quanto aos objetivos das pesquisas selecionadas para análise, observou-se que a maioria visou a avaliar o efeito do aconselhamento farmacêutico aos participantes. $\mathrm{O}$ aconselhamento é uma prática educativa ofertada por profissionais de saúde com o intuito de tornar as pessoas ativas sobre seu processo de saúde, mantendo o respeito à autonomia e valorização de seu potencial, propiciando uma modificação de comportamento e melhoramento na qualidade de vida (FLORES et al., 2018). Segundo a Resolução $n^{\circ}$ 585/13, o aconselhamento é parte das atribuições clínicas do farmacêutico relativas ao cuidado à saúde, nos âmbitos individual e coletivo, e deve conduzir a uma relação de cuidado centrada no paciente (BRASIL, 2013). 
Em relação à idade da população-alvo dos estudos, observou-se que em um estudo a idade das crianças foi de zero até 12 anos. Trata-se de uma das doenças de pele mais corriqueiras no mundo, afetando cerca de $20 \%$ das crianças e $1 \%$ a $3 \%$ dos adultos, o que justifica a maior parte dos estudos encontrados envolver crianças (MAYBA; GOODERHAM, 2017; LEE et al., 2017).

A participação do cuidador mostra a sua relevância como responsável pelo cumprimento do tratamento da DA, possuindo papel central na adesão ao tratamento. $O$ envolvimento dos pais/cuidadores no processo de adesão, mais especificamente no caso de adolescentes, é uma variável importante na adesão e seguimento adequado do tratamento (ZAZULA et al., 2011). Partindo do cuidado em saúde, a DA é pautada no fornecimento de orientações ao paciente. A identificação e o fornecimento das informações corretas disponíveis são desafiadores e podem ser promovidos pela participação de farmacêuticos, que devem desempenhar um papel fundamental na educação do paciente (KOSTER et al., 2019).

Além de orientações sobre a DA, o farmacêutico é essencial na prestação de informações sobre estilo de vida, na escolha do emoliente mais adequado e no tratamento, como no uso de corticosteroides tópicos (KOSTER et al., 2019). Ademais, no estudo de Farrugia et al. (2017) foi percebido que há um grande receio por parte dos cuidadores em sua utilização prolongada, acarretando na falta de adesão. Conforme relatado por Oishi et al. (2019), os farmacêuticos necessitam melhorar seus conhecimentos em relação ao aconselhamento sobre corticosteroides tópicos, pois os pacientes relataram experiências negativas, como visto nos estudos de Farrugia et al. (2017) e de Cheong et al. (2019).

O farmacêutico tem um papel importante na compreensão da doença e do seu tratamento junto aos doentes e dos cuidadores/pais com DA (CORREIA, 2018). O serviço de aconselhamento e educação de DA conduzido por farmacêuticos tem efeito positivo no entendimento dos cuidadores e no gerenciamento da condição do paciente, melhorando a conformidade do tratamento e a qualidade do cuidado (PINHO et al., 2016).

Neste contexto, a comunicação farmacêutico-paciente é crucial para garantir a adesão ao tratamento. Esse profissional deve proporcionar informação compreensiva em relação aos benefícios e riscos do tratamento. A informação inadequada quebra a confiança do paciente, tanto com relação ao farmacêutico quanto no tratamento, gerando medo sobre a utilização dos corticosteroides, o que pode ocasionar a falta de adesão ao tratamento (CARDOSO; PILOTO, 2014).

Esses achados corroboram o resultado de um estudo anterior, que mostra que as intervenções ao paciente melhoram o conhecimento e os resultados clínicos em pacientes com DA (SHIN et al., 2014). Estudos mostraram que intervenções educacionais para pacientes adultos com doenças de pele, incluindo panfletos, palestras e folhetos informativos, foram elaborados em colaboração com especialistas em dermatologia e clínicos gerais, e melhoram os resultados clínicos e o conhecimento sobre cuidados com a pele (ARRUDA; MOREIRA, 2018; HAMMARSTRÖM; WESSLING; NILSSON, 1995).

No que se refere à colaboração multiprofissional, esta tornou-se uma estratégia do trabalho em equipe que atua de forma integrada, compartilhando 
objetivos e colocando os usuários na centralidade do processo, porém consiste em um processo de convivência em um espaço comum entre diferentes profissões que constituem a clínica, envolvendo o processo de comunicação e tomadas de decisão compartilhadas para a melhor produção do cuidado em saúde para seus pacientes (ARRUDA; MOREIRA, 2018; FREIRE et al., 2018).

O trabalho em colaboração traz a interação entre as pessoas, acaba com a individualidade entre os profissionais, contribui para a união e a troca de conhecimento e acarreta em criação de vínculo entre o profissional e o paciente, além de reduzir erros médico e trazer mais segurança ao paciente (UCHÔA et al., 2012). O conhecimento do paciente sobre a DA leva a mudanças comportamentais e subsequentes resultados clínicos (ZAZULA et al., 2011). Quando estes compreendem a doença como doença sem cura, mas que pode ser tratada seguindo as recomendações do profissional de saúde, em particular o farmacêutico, eles visam a buscar pelo melhor tratamento, de acordo com as suas necessidades, usando emolientes para seu tipo de pele, adesão aos corticosteroides tópicos, e, se for necessário, aprendendo sobre a forma correta de aplicação dos medicamentos tópicos. Esses fatores contribuem para que haja melhoria na qualidade de vida do paciente (RODRIGUES, 2016).

Com relação aos componentes das intervenções farmacêuticas elas foram qualificadas em educacional - que visam prestar informações sobre a DA com materiais impresso (folder, cartilhas e panfletos) e a respeito do tratamento farmacológico do paciente; comportamentais - através de dicas alteram as condutas dos pacientes e cuidadores; técnico - fala do tratamento; em relação às estratégias incluem alterações ou modificação da terapia, dosagem e esquema posológico; e combinação usam táticas de mais de uma das 3 categorias citadas (técnicas, educacionais, comportamentais) (CHISHOLM-BURNS et al ., 2010). Os componentes da intervenção focaram na promoção do uso racional de medicamentos e no aconselhamento farmacêutico. Por meio dessas ações o farmacêutico responsabiliza-se pelas necessidades do paciente relacionadas ao medicamento, com o objetivo de obter resultados terapêuticos satisfatórios, buscando a melhoria da qualidade de vida do usuário (PEREIRA; FREITAS, 2008).

Embora a presente pesquisa tenha elucidado quanto ao papel do farmacêutico no cuidado a pacientes com DA, ele possui algumas limitações, destacando-se o pequeno número de estudos disponíveis. Este fato pode estar associado à seleção do idioma, bancos de dados e palavras-chave da pesquisa. Publicações importantes em outros idiomas podem ter sido omitidas pelo uso apenas de palavras-chave em inglês. Da mesma forma, as restrições do banco de dados podem ter impedido a identificação de artigos importantes que não foram indexados nas bases de dados selecionadas. Esta revisão sistemática, entretanto, forneceu evidências de que os farmacêuticos, sozinhos ou como parte integrante de uma equipe multidisciplinar, desempenham um papel fundamental na melhoria dos parâmetros clínicos, humanísticos e econômicos destes pacientes. Assim, esta revisão sintetiza quais cuidados farmacêuticos podem ser ofertados, bem como que estratégias de intervenção podem ser instituídas.

Editora Unijuí - Revista Contexto \& Saúde - ISSN 2176-7114 - v. 21, n. 43, jul./set. 2021 


\section{CONCLUSÃO}

Apesar dos poucos estudos analisados, pode-se observar que o papel do farmacêutico está centralizado no cuidado aos pacientes com DA, prestando serviços de aconselhamento, educação em saúde e contribuindo com informações sobre a doença, sintomas, tratamento farmacológico e não farmacológico e na diminuição de problemas quanto ao uso de medicamentos. Sendo assim, permite que os pacientes e cuidadores compreendam sua condição crônica e melhoria no manejo da DA. Outro aspecto relevante é que na DA as intervenções farmacêuticas possuem um caráter colaborativo, sendo desenvolvidas essencialmente no contexto multiprofissional.

Diante da escassez de pesquisas sobre o tema, destaca-se a importância da realização de mais estudos sobre cuidados farmacêuticos na DA. Recomenda-se que estes estudos priorizem a principal população-alvo da DA (crianças e adolescentes) e tracem estratégias específicas que também envolvam seus cuidadores.

\section{REFERÊNCIAS}

ANTUNES, A. A. et al. Guia prático de atualização em dermatite atópica - Parte I: etiopatogenia, clínica e diagnóstico. Posicionamento conjunto da Associação Brasileira de Alergia e Imunologia e da Sociedade Brasileira de Pediatria. Arquivos de Asma, Alergia e Imunologia, v. 1, n. 2, p. 131-156, 2017.

ARRUDA, L. S.; MOREIRA, C. O. F. Colaboração interprofissional: um estudo de caso sobre os profissionais do Núcleo de Atenção ao Idoso da Universidade Estadual do Rio de Janeiro (NAI/Uerj), Brasil. Interface-Comunicação, Saúde, Educação, v. 22, p. 199-210, 2018.

BRASIL. Conselho Federal de Farmácia. Resolução no 585, de 29 de agosto de 2013, que regulamenta as atribuições clínicas do farmacêutico e dá outras providências. Diário Oficial da União, Poder Executivo, Brasília, DF, 2013. Disponível em: htts://www.cff.org.br/ userfiles/file/noticias/Resolu\%C3\%A7\%C3\%A30586_13.pdf. Acesso em: 12 dez. 2019.

BRASIL. Ministério da Saúde. Cuidado farmacêutico na atenção básica; caderno 2. 2014. Disponível em: http://bvsms.saude.gov.br/bvs/publicacoes/cuidado_farmaceutico_atencao_basica_saude_2.pdf. Acesso em: 12 dez. 2019.

CARDOSO, D. M.; PILOTO, J. A. R. Atenção farmacêutica ao idoso: uma revisão. Brazilian Journal of Surgery and Clinical Research., v. 9, n. 1, p. 60-66, 2014.

CARVALHO, V. O. et al. Guia prático de atualização em dermatite atópica - Parte II: abordagem terapêutica. Posicionamento conjunto da Associação Brasileira de Alergia e Imunologia e da Sociedade Brasileira de Pediatria. Arquivos de Asma Alergia e Imunologia, v. 1, n. 2, 2017.

CHEONG, J. Y. V. et al. Impact of pharmacists' counseling on caregiver's knowledge in the management of pediatric atopic dermatitis. Pediatric Dermatol., v. 36, ed. 1, p. 105-109, 2019.

CHISHOLM-BURNS, M. A. et al. US pharmacists' effect as team members on patient care: systematic review and meta-analyses. Med Care, v.48, n. 10, p. 923-33, 2010.

CORREIA, K. K. L. et al. Farmácia clínica: importância deste serviço no cuidado a saúde. Boletim Informativo Geum, v. 8, n. 3, p. 7, 2018.

FARRUGIA, L. L. et al. Evaluation of the influence of pharmacists and GPs on patient perceptions of long-term topical corticosteroid use. Journal of Dermatological Treatment, v. 28, n. 2, p. 112-118, 2017.

Editora Unijuí - Revista Contexto \& Saúde - ISSN 2176-7114 - v. 21, n. 43, jul./set. 2021 
FLORES, T. R. et al. Aconselhamento por profissionais de saúde e comportamentos saudáveis entre idosos: estudo de base populacional em Pelotas, sul do Brasil, 2014. Epidemiologia e Serviços de Saúde, Brasília. v. 27, n. 1, p. 1-11, 2018.

FREIRE, J. R. F. et al. Atitudes para a colaboração interprofissional de equipes da Atenção Primária participantes do Programa Mais Médicos. Revista Latino-Americana de Enfermagem, Ribeirão Preto, v. 26, 2018.

GALVÃO, T. F.; PANSANI, T. S. A.; HARRAD, D. Principais itens para relatar revisões sistemáticas e meta-análises: a recomendação Prisma. Epidemiologia e Serviços de Saúde, v. 24, p. 335-342, 2015.

GUILHERME, A. Q. et al. Abordagem da dermatite atópica na infância pelo médico de família e comunidade. Revista Brasileira Med. Fam. Comunidade, v. 9, n. 31, p. 159-68, 2014.

HAMMARSTRÖM, B.; WESSLING, A.; NILSSON, J. L. Pharmaceutical care for patients with skin diseases: a campaign year at Swedish pharmacies. Journal of Clinical Pharmacy and Therapeutics, v. 20, n. 6, p. 327-334, 1995.

KOSTER, E. S. et al. Optimizing pharmaceutical care for pediatric patients with dermatitis: perspectives of parents and pharmacy staff. International Journal of Clinical Pharmacy, p. 1-8, 2019.

LEE, J. et al. Is the prevalence of atopic dermatitis in korean children decreasing? Analysis of the national statistics data, 2009-2014. Asian. Pac. J. Allergy Immunol, v. 35, p. 144149, 2017.

LIMA, Andréia Carnib Bemvindo; NUNES, Ivone Freires de Oliveira Costa. O papel da vitamina D na dermatite atópica. Journal of Health Sciences, v. 17, n. 4, 2015.

MAYBA, J. N.; GOODERHAM, Review of Atopic Dermatitis and Topical Therapies. Journal of Cutaneous Medicine and Surgery, v. 21, n. 3, p. 227-236, 2017.

MENDONÇA, L. G.; FERREIRA, F. R.; LAROCQUE, L. R. Trajetória da educação farmacêutica e o lugar da deontologia e ética na formação humanista: uma discussão curricular. Currículo Sem Fronteiras, Rio de Janeiro, v. 17, n. 2, p.458-484, 2017.

MENEGUIN, S.; RIBEIRO, R. Dificuldades de cuidadores de pacientes em cuidados paliativos na estratégia da saúde da família. Texto Contexto - Enferm., Florianópolis, v. 25, n. $1,2016$.

OISHI, N. et al. A survey on awareness of the" finger-tip unit" and medication guidance for the use of topical steroids among community pharmacists. Drug Discoveries \& Therapeutics, v. 13, n. 3, p. 128-13, 2019.

PEREIRA, L. R. L.; FREITAS, O. A evolução da Atenção Farmacêutica e a perspectiva para o Brasil. Revista Brasileira de Ciências Farmacêuticas, v. 44, n. 4, p. 601-12, 2008.

PINHO, M. S. et al. Atenção farmacêutica a pacientes oncológicos: uma revisão integrativa da literatura. Revista Brasileira de Farmácia Hospitalar e Serviços de Saúde, v. 7, n. 1, 2016.

RODRIGUES, A. M. F. Uma aproximação farmacêutica à dermatite atópica. 2016. Monografia - Faculdade de Farmácia da Universidade de Coimbra. Disponível em: https:// estudogeral.uc.pt/handle/10316/42828. Acesso em: 25 jun. 2019.

SBD. Sociedade Brasileira de Dermatologia. Eczema. Disponível em: https://www.sbd.org. br/dermatologia/pele/doencas-e-problemas/eczema/70/. Acesso em: 13 dez. 2019.

SHIN, J. Y. et al. Um programa educacional que contribui para melhorar a compreensão dos pacientes e dos pais sobre a dermatite atópica. Ann Dermatol., v. 26, n. 1, p. 66-72, 2014.

TUCKER, R. P. et al. An exploratory study of community pharmacist diagnosis and management of dermatitis and acne. Selfcare, v. 8, n. 2, p. 1-10, 2017.

UCHÔA, A. C. et al. Trabalho em equipe no contexto da reabilitação infantil. Physis, Rio de Janeiro, v. 22, n. 1, p. 385-400, 2012.

Editora Unijuí - Revista Contexto \& Saúde - ISSN 2176-7114 - v. 21, n. 43, jul./set. 2021 
WONG, I. T. Y. et al. Guidelines for the management of atopic dermatitis (eczema) for pharmacists. Canadian Pharmacists Journal/Revue des Pharmaciens du Canada, v. 150, n. 5, p. 285-297, 2017.

YAMADA, K. N. T.; NABESHIMA, T. Pharmacist-managed clinics for patient education and counseling in Japan: current status and future perspectives. J Pharm Health Care Sci., v. 1, n. 2, 2015.

ZAZULA, R. et al. Educação terapêutica para a pacientes com dermatite atópica e seus cuidadores: uma revisão sistemática. Acta Comport., Guadalajara, v. 19, n. 2, p. 241-252, 2011.

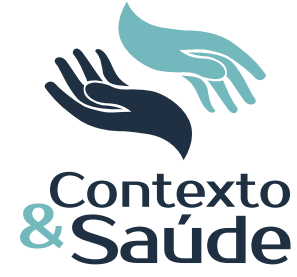

